\title{
Cosmic string in the van Stockum cylinder
}

\author{
J P Krisch \\ Department of Physics, University of Michigan, Ann Arbor, MI 48109, USA
}

Received 18 October 2002

Published 7 April 2003

Online at stacks.iop.org/CQG/20/1605

\begin{abstract}
The low density van Stockum dust solution is extended by including an angular deficit factor. The resulting model describes a rotating Gott-Hiscock string surrounded by an annular dust atmosphere. The interior spacetime can be joined to a vacuum Levi-Civita solution with angular deficit.
\end{abstract}

PACS numbers: $04.20 . J b, 04.40 .-b, 98.80 . \mathrm{Hw}$

\section{Introduction}

Describing the interior and exterior geometries of spacetimes with cylindrical symmetry has a long history in general relativity. Recently there has been a renewed interest [1-9], in the Levi-Civita [10] spacetime

$$
\mathrm{d} s^{2}=-r^{2 m} \mathrm{~d} \tau^{2}+r^{2 m^{2}-2 m}\left(\mathrm{~d} r^{2}+\mathrm{d} z^{2}\right)+C^{2} r^{2-2 m} \mathrm{~d} \phi^{2} .
$$

The metric describes a vacuum with cylindrical symmetry and is often used to match cylindrical matter distributions. One reason for the current interest in this spacetime is the problem in identifying sources that cover the entire range of the Levi-Civita parameters. This difficulty has been reviewed by Bonnor [11]. In different parts of its parameter range, the Levi-Civita spacetime is the vacuum solution for two very interesting cylindrical solutions. One of the early rotating matter solutions, describing an infinite cylinder of rotating dust, was found by Lanczos [12] and van Stockum [13] and later by Wright [14]. The vacuum spacetime matching the rotating dust interior was discussed by Lewis [15], van Stockum [13] and Bonnor [16]. It takes one of three forms depending on the mass density of the cylinder. The vacuum corresponding to the lowest range of rotating cylinder mass density is locally static and can be transformed into a Levi-Civita spacetime $[17,18]$ with the parameter $C=1$. The second solution was developed by Hiscock [19] and Gott [20] and describes the static interior of an infinite cosmic string. The spacetime of the string interior can be matched to an $m=0$ Levi-Civita solution with $C \neq 1$. This Levi-Civita vacuum has the string trademark angular deficit.

In this paper, we re-examine the solution for the rotating dust interior in the lowest mass density region. In the original discussions, several constants were chosen to be zero or one. If the constants are kept, three new features appear. The first new feature is an angular deficit 
in the locally static Levi-Civita form of the exterior vacuum, $C \neq 1$. The addition of the constants adds to the sources that can be described by the Levi-Civita parameter range. The presence of angular deficit suggests a string structure interior to the dust and the second new feature is a rotating core solution. In the limit of zero rotation the solution is the static spacetime describing the Gott-Hiscock [19, 20], cosmic string. The new van Stockum constants can be linked to the string angular deficit through matching. If the axial rotating string is not present, the dust cylinder will develop closed timelike lines in the interior. This third new feature offers another venue for the study of closed timelike lines in the presence of matter.

In the following section we develop the extended interior dust solution. The spinning Gott-Hiscock $[19,20]$ string is discussed in section 3. The locally static form of the vacuum with angular deficit is treated in section 4 . A discussion of the closed timelike lines is given in the concluding section.

\section{The interior field equations}

\section{1. van Stockum matter content}

Consider a stationary metric of the van Stockum type

$$
\mathrm{d} s^{2}=-(\mathrm{d} t+k(r) \mathrm{d} \phi)^{2}+\mathrm{e}^{\mu(r)}\left(\mathrm{d} r^{2}+\mathrm{d} z^{2}\right)+D^{2}(r) \mathrm{d} \phi^{2} .
$$

The field equations with an anisotropic fluid source for this metric are

$\mathrm{e}^{-\mu}\left(\frac{k, r}{2 D}\right), r=0$

$$
\begin{array}{ll}
8 \pi \rho \mathrm{e}^{\mu}=\frac{3 k,_{r}^{2}}{4 D^{2}}-\frac{\mu,_{r}, r}{2}-\frac{D,_{r}, r}{D} & 8 \pi p_{r} \mathrm{e}^{\mu}=\frac{k,_{r}^{2}}{4 D^{2}}+\frac{D,_{r}}{D} \frac{\mu,_{r}}{2} \\
8 \pi p_{\phi} \mathrm{e}^{\mu}=\frac{k,_{r}^{2}}{4 D^{2}}+\frac{\mu,_{r}, r}{2} & 8 \pi p_{z} \mathrm{e}^{\mu}=-\frac{k,_{r}^{2}}{4 D^{2}}-\frac{D,_{r}}{D} \frac{\mu,_{r}}{2}+\frac{D,_{r}, r}{D} .
\end{array}
$$

Requiring isotropic dust, we find $D,{ }_{r}, r=0$. The most general solution is $D(r)=$ $d_{0} r+d_{1}$. Choosing $d_{1}=0$, we find for the interior potentials and matter density

$$
\begin{array}{ll}
D(r)=d_{0} r & k(r)=\omega d_{0} r^{2}+c_{1} \\
\mu(r)=-\omega^{2} r^{2}+c_{2} & 8 \pi \rho \mathrm{e}^{\mu}=4 \omega^{2} .
\end{array}
$$

$\omega$ is a constant identified from the original van Stockum solution. The original choice [12-14] was $c_{1}=c_{2}=0, d_{0}=1, d_{1}=0$. The $d_{0}$ factor cannot be scaled away by redefining $\varphi$, but signals the presence of an angular deficit in $\varphi$. Angular deficits are commonly associated with axial strings. In the following section we develop a core string solution inside the dust cylinder. $c_{1}$ and $d_{0}$ are independent but will be related when the van Stockum dust becomes the annular atmosphere for the rotating string.

\section{A core metric}

\subsection{Describing the core}

The Gott-Hiscock [19, 20] static string describes the interior spacetime of a string with constant density, $\varepsilon$, axial tension $T=\varepsilon$ and no other stresses. To describe a rotating string, assume an interior structure described by coordinates $(t, \chi, \psi, \xi)$ with boundary $\chi=\chi_{0}$. 
The core spacetime will have a $\xi$ axial tension, $T_{0}^{2}$ and no radial stress. With the change in coordinates and stress energy content, the field equations are repeated for clarity

$$
\begin{aligned}
& k,_{\chi}=\lambda D(\chi) \\
& 8 \pi \rho \mathrm{e}^{\mu}=\frac{3 \lambda^{2}}{4}-\frac{\mu, \chi, \chi}{2}-\frac{D,_{\chi}, \chi}{D} \\
& 8 \pi p_{\chi} \mathrm{e}^{\mu}=\frac{\lambda^{2}}{4}+\frac{\mu,_{\chi}}{2} \frac{D,_{\chi}}{D}=0 \\
& 8 \pi p_{\psi} \mathrm{e}^{\mu}=\frac{\lambda^{2}}{4}+\frac{\mu,,_{\chi}}{2} \\
& 8 \pi p_{\xi} \mathrm{e}^{\mu}=-\frac{\lambda^{2}}{4}-\frac{\mu,_{\chi}}{2} \frac{D,_{\chi}}{D}+\frac{D,_{\chi}, \chi}{D}=-T_{0}^{2},
\end{aligned}
$$

where $\lambda$ is a constant. The $\xi$-field equations determine $D(\chi)$ and from that $\mu(\chi)$ and $k(\chi)$ are found. Assuming a Minkowski axis, the solutions are

$D(\chi)=\frac{\sin \left(T_{0} \chi\right)}{T_{0}} \quad \mathrm{e}^{\mu(\chi)}=\left[\cos \left(T_{0} \chi\right)\right]^{\lambda^{2} / 2 T_{0}^{2}} \quad k(\chi)=\frac{\lambda}{\left(T_{0}\right)^{2}}\left(1-\cos \left(T_{0} \chi\right)\right)$.

The fluid parameters associated with this solution are

$8 \pi p_{\psi}=-\frac{\lambda^{2} \mathrm{e}^{-\mu}}{4} \tan ^{2}\left(T_{0} \chi\right) \quad 8 \pi \rho=\mathrm{e}^{-\mu}\left(\frac{3 \lambda^{2}}{4}+T_{0}^{2}+\frac{\lambda^{2}}{4} \sec ^{2}\left(T_{0} \chi\right)\right)$.

Because of the trigonometric structure of the matter descriptions there is a constraint for well-behaved solutions

$$
T_{0} \chi<\frac{\pi}{2}
$$

In the limit of zero rotation, $\lambda \longrightarrow 0$, the solution becomes the Gott-Hiscock $[19,20]$ static string with equation of state $8 \pi \rho=-8 \pi p_{\xi}=T_{0}^{2}$.

\subsection{Matching the string to dust}

The metric and extrinsic curvatures of the dust and string are matched across the bounding surface $\left(\chi=\chi_{1}, r=R_{1}\right)$. It proceeds exactly as in the vacuum match for the Gott-Hiscock string $[19,20]$. Matching the $D$ functions we have

$$
\sin \left(T_{0} \chi_{1}\right)=d_{0} T_{0} R_{1} \quad \cos \left(T_{0} \chi_{1}\right)=d_{0} .
$$

Relating the tension to $d_{0}$ and $R_{1}$ we find

$$
T_{0}^{2} R_{1}^{2}=\frac{1-d_{0}^{2}}{d_{0}^{2}}
$$

This implies that $d_{0} \leqslant 1$. This relation is very interesting. In a later section we will demonstrate that the angular deficit in the locally flat vacuum is related to $d_{0}$. Here we see that $d_{0}$ is related to the sizes of the tension and inner radius. Matching the off-diagonal $g_{i j}$ provides the relation

$$
\frac{\lambda}{T_{0}^{2}}\left(1-\cos \left(T_{0} \chi_{1}\right)\right)=\omega d_{0} R_{1}^{2}+c_{1} \quad \frac{\lambda}{T_{0}} \sin \left(T_{0} \chi_{1}\right)=2 \omega d_{0} R_{1} .
$$

Combining the equations we identify $\lambda$ and $c_{1}$

$$
\lambda=2 \omega \quad c_{1}=\omega d_{0} R_{1}^{2} \frac{d_{0}-1}{d_{0}+1} .
$$


Since $d_{0} \leqslant 1$, the last equation requires $c_{1} \leqslant 0$. This relation links $c_{1}$ and $d_{0} . d_{0}$, representing angular deficit, cannot be scaled away and through matching, $c_{1}$ is also now a necessary part of the metric. The final match is in the coordinates $\xi$ and $z$. It duplicates $\lambda=2 \omega$ and sets a value for $c_{2}$

$$
c_{2}=\omega^{2} R_{1}^{2}\left(1+\frac{2 d_{0}^{2}}{1-d_{0}^{2}} \ln \left(d_{0}\right)\right) .
$$

The interior solution can be described as a string-like structure with axial tension and tension loops around the circumference of the inner core cylinder. The core is surrounded by an annular atmospheric region of dust. The dust can be matched to a vacuum and this is where the final angular deficit will appear.

\section{The vacuum region}

The annular dust atmosphere is bounded by a vacuum at $r=R_{2}$. The vacuum field equations have been written down by van Stockum [13] and Bonnor [16] and discussed in detail by Islam [21] for the general metric

$$
\mathrm{d} s^{2}=-f_{v}\left(\mathrm{~d} t+\frac{k_{v}}{f_{v}} \mathrm{~d} \phi\right)^{2}+\mathrm{e}^{\mu_{v}}\left(\mathrm{~d} r^{2}+\mathrm{d} z^{2}\right)+\frac{D_{v}^{2}}{f_{v}} \mathrm{~d} \phi^{2} .
$$

We use $D_{v}=d_{0} r$ which produces a slight modification in the form of the vacuum solution used by Bonnor. For $r \geqslant R_{2}$ the vacuum solution with unmatched constants is

$$
\begin{aligned}
& D_{v}(r)=d_{0} r \\
& f_{v}(r)=D_{v}(r)(\alpha \sinh U+\beta \cosh U) \\
& k_{v}(r)=D_{v}(r)(\gamma \sinh U+\delta \cosh U) \\
& U=A \ln \left(\frac{r}{R_{2}}\right) \\
& \mu_{v}(r)=\mu_{1}+\frac{A^{2}-1}{2} \ln \left(\frac{r}{R_{2}}\right) .
\end{aligned}
$$

$A$ is an undetermined constant. In the interior of the annular dust region, $r \leqslant R_{2}$, the metric potentials are

$$
\begin{aligned}
& D(r)=d_{0} r \\
& f=1 \quad k(r)=\omega d_{0} r^{2}+c_{1} \quad \mu(r)=-\omega^{2} r^{2}+c_{2} .
\end{aligned}
$$

Matching $f$ and $f_{v}$ one finds

$$
d_{0} R_{2} \beta=1 \quad \frac{1}{d_{0} R_{2}}+A \alpha=0 .
$$

From the $k, k_{v}$ match we have

$$
\delta=\omega R_{2}+\frac{c_{1}}{d_{0} R_{2}} \quad A \gamma=\omega R_{2}-\frac{c_{1}}{d_{0} R_{2}} .
$$

Matching $\mu$ and $\mu_{v}$ at $r=R_{2}$, we find that the constants $\mu_{1}$ and $A$ are both identified

$$
\mu_{1}=-\omega^{2} R_{2}^{2}+c_{2} \quad A^{2}=1-4 \omega^{2} R_{2}^{2} .
$$


The complete vacuum solution including the effects of $d_{0}, c_{1}$ and $c_{2}$ is

$$
\begin{aligned}
& \mathrm{e}^{\mu_{v}}=\mathrm{e}^{-\omega^{2} R_{2}^{2}+c_{2}}\left(\frac{R_{2}}{r}\right)^{2 \omega^{2} R_{2}^{2}} \quad f_{v}=\frac{r}{R_{2}}\left(\cosh U-\frac{\sinh U}{A}\right) \\
& k_{v}=d_{0} r\left(\frac{\omega R_{2}-\frac{c_{1}}{d_{0} R_{2}}}{A} \sinh U+\left(\omega R_{2}+\frac{c_{1}}{d_{0} R_{2}}\right) \cosh U\right) \\
& A^{2}=1-4 \omega^{2} R_{2}^{2} \quad D_{v}=d_{0} r .
\end{aligned}
$$

The original Bonnor [16] vacuum matched to dust is

$$
\begin{aligned}
\mathrm{e}^{\mu_{B}} & =\mathrm{e}^{-\omega^{2} R_{2}^{2}}\left(\frac{R_{2}}{r}\right)^{2 \omega^{2} R_{2}^{2}} \quad f_{B}=\frac{r}{R_{2}}\left(\cosh U-\frac{\sinh U}{A}\right) \\
k_{B} & =r\left(\frac{\omega R_{2}}{A} \sinh U+\omega R_{2} \cosh U\right) \\
A^{2} & =1-4 \omega^{2} R_{2}^{2} \quad D_{B}=r .
\end{aligned}
$$

The new constants do not change $g_{00}$. The off-diagonal metric does change with the original Bonnor solution recovered in the $d_{0} \rightarrow 1, c_{1} \rightarrow 0$ limit. $\mathrm{e}^{\mu_{v}}$ is an overall constant different from the original value. The Bonnor vacuum can be transformed into a locally static Levi-Civita form with no angular deficit. The new solution will exhibit an angular deficit.

\section{The locally static form for $\omega R_{2} \leqslant 1 / 2$}

Bonnor [16] has shown that the spacetime described by equation (28) is locally static with a periodic time coordinate. The transformation to the locally static case has also been discussed by Frehland [17] and Som [18]. We wish to check the effect of adding the new constants on the locally static form. To do this we will consider a coordinate transformation of the spacetime of equation (17). Consider the transformation

$$
t=a \tau+c \theta \quad \varphi=b \tau+h \theta,
$$

with $a h-b c=1$. With this transformation, the spacetime becomes

$\mathrm{d} s^{2}=-f_{v}\left[(a \mathrm{~d} \tau+c \mathrm{~d} \theta)+\frac{k_{v}}{f_{v}}(b \mathrm{~d} \tau+h \mathrm{~d} \theta)\right]^{2}+\mathrm{e}^{\mu_{v}}\left(\mathrm{~d} r^{2}+\mathrm{d} z^{2}\right)+\frac{D_{v}^{2}}{f_{v}}(b \mathrm{~d} \tau+h \mathrm{~d} \theta)^{2}$.

Use the forms of $f_{v}(r)$ and $k_{v}(r)$, equation (19) before matching. Examining first the off-diagonal component and requiring it to vanish one finds

$$
a=-b \frac{\gamma+\delta}{\alpha+\beta} \quad c=-h \frac{\delta-\gamma}{\beta-\alpha},
$$

with the conditions

$$
\begin{aligned}
& \alpha^{2}-\beta^{2}=(\beta \gamma-\delta \alpha)^{2} \\
& b^{2} h^{2}=\frac{\alpha^{2}-\beta^{2}}{4} .
\end{aligned}
$$

Equation (32) only restates equation (26), determining the value of $A$. The last condition follows from the unit transformation determinant. With these choices, the metric is

$$
g_{00}=-b^{2}\left(\frac{-2 d_{0} R_{2}^{A}}{\alpha+\beta}\right) r^{1-A}
$$




$$
g_{\theta \theta}=h^{2} \frac{2 d_{0}}{\beta-\alpha} r^{1+A} \quad g_{r r}=\mathrm{e}^{\left(A^{2}-1\right) / 4} \mathrm{e}^{c_{2}}\left(\frac{R_{2}}{r}\right)^{\left(1-A^{2}\right) / 2} .
$$

In the original formulation, with $c_{2}=0, d_{0}=1$, new $r$ and $z$ coordinates can be defined as

$$
r^{\prime}=\Sigma r \quad z^{\prime}=\Sigma z \quad \Sigma=\frac{\mathrm{e}^{\left(A^{2}-1\right) / 2\left(A^{2}+3\right)}}{R_{2}^{\left(A^{2}-1\right) /\left(A^{2}+3\right)}} .
$$

This moves the $g_{r r}$ and $g_{z z}$ parts of the metric into the Levi-Civita form and $g_{00}$ becomes

$$
g_{00}=-\left(r^{\prime}\right)^{1-A} b^{2}\left(\frac{-2 R_{2}^{A}}{\alpha+\beta}\right) \Sigma^{A-1} .
$$

If we again choose a Levi-Civita form for $g_{00}$ by setting

$$
b^{2}=-\frac{\alpha+\beta}{2 \Sigma^{A-1} R_{2}^{A}},
$$

the form for $g_{\theta \theta}$ is also determined and we find for the metric

$$
\mathrm{d} s^{2}=-\left(r^{\prime}\right)^{1-A} \mathrm{~d} \tau^{2}+\frac{\left(r^{\prime}\right)^{1+A}}{\Sigma^{2}} \mathrm{~d} \theta^{2}+\left(r^{\prime}\right)^{\left(A^{2}-1\right) / 2}\left(\mathrm{~d} r^{\prime 2}+\mathrm{d} z^{\prime 2}\right) .
$$

An angular deficit can be calculated by comparing the limiting ratio of the circumference to the proper radius [22]

$$
\delta \theta=2 \pi-\lim _{R_{p} \rightarrow 0} \frac{\text { circumference }}{\text { proper radius }} .
$$

The ratio of the circumference to the radius for this spacetime with $d_{0}=1, c_{2}=0$ is

$$
\frac{C}{R_{p}}=\frac{2 \pi R_{2}^{(1+A) / 2}}{R_{2}^{\left(A^{2}+3\right) / 4}} \frac{A^{2}+3}{4 \Sigma} .
$$

In the limit that the proper radius (or $R_{2}$ ) approaches zero, $A \rightarrow 1, \Sigma \rightarrow 1$ and this ratio is just $2 \pi$. There is no angular deficit in the original formulation. With the new constants we can follow the same procedure. There are two differences: the redefinition of the $r$ and $z$ coordinates acquires a factor from $c_{2}$ and $d_{0}^{2}$ appears in $g_{\theta \theta}$. Defining $\Sigma^{\prime}$,

$$
\Sigma^{\prime}=\Sigma \mathrm{e}^{2 c_{2} /\left(A^{2}+3\right)},
$$

the new spacetime becomes

$$
\mathrm{d} s^{2}=-\left(r^{\prime}\right)^{1-A} \mathrm{~d} \tau^{2}+\frac{\left(r^{\prime}\right)^{1+A} \mathrm{~d}_{0}^{2}}{\Sigma^{\prime 2}} \mathrm{~d} \theta^{2}+\left(r^{\prime}\right)^{\left(A^{2}-1\right) / 2}\left(\mathrm{~d} r^{\prime 2}+\mathrm{d} z^{\prime 2}\right) .
$$

The only new thing to examine is the behaviour of $c_{2}$. From equation (16) we have $c_{2}=\omega^{2} R_{1}^{2}\left(1+2 \frac{d_{0}^{2}}{1-d_{0}^{2}} \ln \left(d_{0}\right)\right)$. As the $R_{2}$ limit is taken, $R_{1}$ should also approach zero and $c_{2} \rightarrow 0$. The overall limit gives an angular deficit in $\theta$

$$
\delta \theta=2 \pi\left(1-d_{0}\right) .
$$

$d_{0}$ is related to the axial tension and in the limit of small $T_{0} R_{1}$ we have $d_{0} \approx 1-\frac{T_{0}^{2} R_{1}^{2}}{2}$ and the angular deficit is

$$
\delta \theta \approx \pi T_{0}^{2} R_{1}^{2} .
$$

In the zero rotation limit, the tension and density have a string equation of state and $d_{0}=1-4 \pi \rho R_{1}^{2}$. In this limit, the linear mass density of the string, $m / L$, is $\left(1-d_{0}\right) / 4$ and the angular deficit is simply the Vilenkin [23] value of $8 \pi \mathrm{m} / L$. 


\section{Conclusion}

The new spinning string solution with an annular dust atmosphere links a rotating GottHiscock [19, 20] spacetime and an extended van Stockum [13] solution. Together they provide a source for a locally static vacuum Levi-Civita spacetime with angular deficit. The original van Stockum solution has been of interest because closed timelike lines [24] can develop in the high density region of the vacuum exterior and it, along with the Kerr [25], Godel [26] and other solutions [27], has provided a means to study this effect. Adding angular deficit to the van Stockum dust interior could also create closed timelike lines in the interior spacetime. The metric potential $g_{\phi \phi}$ of the extended dust solution is

$$
g_{\phi \phi}=d_{0}^{2} r^{2}-\left(\omega d_{0} r^{2}+c_{1}\right)^{2} \geqslant 0 .
$$

Closed timelike lines will develop inside the cylinder if $g_{\phi \phi}<0$. Requiring the interior $g_{\phi \phi} \geqslant 0$, we see that there is a lower limit on the radial coordinate, below which closed timelike lines will develop

$$
1-2 \omega \frac{c_{1}}{d_{0}}-\sqrt{1-4 \omega \frac{c_{1}}{d_{0}}} \leqslant 2 \omega^{2} r^{2} \leqslant 1-2 \omega \frac{c_{1}}{d_{0}}+\sqrt{1-4 \omega \frac{c_{1}}{d_{0}}} .
$$

The original van Stockum solution $\left(c_{1}=0, d_{0}=1\right)$ had limits $0 \leqslant \omega^{2} r^{2} \leqslant 1$. As van Stockum [2] pointed out, this is just the special relativistic limit on velocity, although $r$ in this case is not a proper radius. There are no closed timelike lines in the van Stockum dust interior. When $c_{1} \neq 0$, there can be interior closed timelike lines if the dust extends to the centre. If the van Stockum dust is the only matter in the cylinder, if there is no string core, then, $c_{1}$ and $d_{0}$ are independent. The extended van Stockum solution, by itself, then has new effects in the low density region associated with the constants $d_{0}$ and $c_{1}$ : closed timelike lines associated with $c_{1}$ develop in the dust interior and there is a vacuum angular deficit associated with $d_{0}$. If there is a cosmic string interior to the rotating dust, the constants $c_{1}$ and $d_{0}$ are related by the string-dust matching. The dust in the model discussed in this paper is in an annular region $R_{1} \leqslant r \leqslant R_{2}$. A particular choice of $R_{1}$ could either eliminate or include the interior closed timelike lines. Bonnor [27] has recently stressed the importance of developing a better understanding of closed timelike lines and this solution offers another venue for their study.

\section{References}

[1] Stachel J 1982 Phys. Rev. D 261281

[2] Bonnor W B and Davidson W 1992 Class. Quantum Grav. 92065

[3] Philbin T G 1996 Class. Quantum Grav. 131217

[4] Wang A Z, da Silva M F A and Santos N O 1997 Class. Quantum Grav. 142417

[5] Herrera L, Paiva Filipe M and Santos N O 1999 J. Math. Phys. 404064

[6] da Silva M F A, Wang A, Paiva Filipe M and Santos N O 2000 Phys. Rev. D 61044003

[7] Herrera L, Santos N O, Teizeira A F F and Wang A Z 2001 Class. Quantum Grav. 183847

[8] Miguelote A Y, da Silva M F A, Wang A and Santos N O 2001 Class. Quantum Grav. 184569

[9] Bicak J and Zofka M 2002 Class. Quantum Grav. 193653

[10] Levi Civita T 1917 Rend. Acc. Liniei 26307

[11] Bonnor W B 1999 The static cylinder in general relativity On Einstein's Path: Essays in Honor of Engelbert Schucking ed A Harvey (New York: Springer)

[12] Lanczos K 1997 Gen. Rel. Grav. 29363

[13] van Stockum W J 1937 Proc. R. Soc. Edinburgh 57135

[14] Wright J P 1965 J. Math. Phys. 6103

[15] Lewis T 1932 Proc. R. Soc. A 136176

[16] Bonnor W B 1980 J. Phys. A: Math. Gen. 132121 
[17] Frehland E 1971 Commun. Math. Phys 23127

[18] Som M M, Teizeira A F F and Wolk I 1976 Gen. Rel. Grav. 7263

[19] Hiscock W A 1985 Phys. Rev. 313288

[20] Gott J R III 1985 Astrophys. J. 288422

[21] Islam J N 1985 Rotating Fields in General Relativity (Cambridge: Cambridge University Press)

[22] Frolov V P, Israel W and Unruh W G 1989 Phys. Rev. D 391084

[23] Vilenkin A 1981 Phys. Rev. D 23352

[24] Tipler F J 1974 Phys. Rev. D 92203

[25] Kerr R P 1963 Phys. Rev. Lett. 11237

[26] Godel K 1949 Rev. Mod. Phys. 21447

[27] Bonnor W B 2002 Closed timelike curves in general relativity Preprint gr-qc/0211051 